\title{
A Phase II study of celecoxib, gemcitabine, and cisplatin in advanced pancreatic cancer
}

\author{
Basil F. El-Rayes ${ }^{1}$, Mark M. Zalupski ${ }^{2}$, Anthony F. Shields ${ }^{1}$, Ann Marie Ferris ${ }^{1}$, Ulka Vaishampayan ${ }^{1}$, \\ Lance K. Heilbrun ${ }^{1}$, Raghu Venkatramanamoorthy ${ }^{1}$, Volkan Adsay ${ }^{1}$ and Philip A. Philip ${ }^{1}$ \\ ${ }^{1}$ Karmanos Cancer Institute, Wayne State University, Detroit, Michigan; ${ }^{2}$ University of Michigan, Ann Arbor, Michigan
}

Key words: pancreatic cancer, cisplatin, celecoxib, gemcitabine

\begin{abstract}
Summary
Background. Pancreatic cancer is amongst the most chemoresistant malignancies. Expression of the cyclooxygenase-2 (COX-2) enzyme plays a major role in tumor progression and resistance to therapy. A Phase II study was undertaken to determine the effect of gemcitabine by fixed-dose rate infusion (FDR), cisplatin and the COX-2 inhibitor, celecoxib, on the 6-month survival rate in patients with metastatic pancreatic cancer.

Methods. The eligibility criteria included a pathologically or cytologically confirmed diagnosis of adenocarcinoma of the pancreas. No prior gemcitabine therapy was allowed. Patients received a combination of gemcitabine $1000 \mathrm{mg} / \mathrm{m}^{2}$ over 100 minutes, cisplatin $35 \mathrm{mg} / \mathrm{m}^{2} \mathrm{I}$.V. on days 1 and 8, and celecoxib continuously at a daily dose of $800 \mathrm{mg}$. Cycles were repeated every 21 days.

Results. Twenty-two patients with metastatic pancreas cancer were enrolled (median age, 59.5 years; M:F, 13:9). The median number of cycles was 2 per patient. The median survival time was 5.8 months (90\% CI, 3.6-7.6 months). The probability of survival at 6 months was $46 \%(90 \%$ CI, 27-62\%). The major toxicity was neutropenia with grade 3 or 4 toxicities seen in $65 \%$ of patients.

Conclusions. The addition of celecoxib to gemcitabine (by FDR) and cisplatin did not appear to increase activity of the chemotherapy doublet in patients with advanced pancreatic cancer. Celecoxib alone may not be sufficient to sensitize pancreatic cancer to the effects of conventional cytotoxic therapy.
\end{abstract}

\section{Introduction}

Pancreatic cancer is the fourth leading cause of cancer death in the United States [1]. Despite an increase in the number of systemic treatments available for pancreatic cancer, the impact of therapy on the clinical course of this disease has been modest [2]. The chemoresistant nature of pancreatic cancer cells have limited the development of effective therapies. Therefore, improvements in the outcome of patients with pancreatic cancer are very much dependent on the development of more effective systemic agents with novel mechanisms of action.

The cyclooxygenase (COX) isoenzymes catalyze the rate-limiting step in the conversion of arachidonic acid into prostaglandins [3]. Recent evidence suggests that COX-2 has a central role in pancreatic carcinogenesis and

This study was supported in part by Cancer Center Support Grant CA22453 from the National Cancer Institute and by Eli Lilly Oncology. in the chemoresistance of established disease [4]. Work from our institution and others showed that the COX2 isoenzyme is overexpressed in $45-75 \%$ of pancreatic cancer $[5,6]$ and has been associated with increased invasiveness [7] and promotion of angiogenesis [8] Preclinical evidence suggested that the COX-2 mRNA is stabilized by mutations in the $k$-ras oncogene that are very frequently present in pancreas cancer $[9,10]$. Selective and nonselective COX-2 inhibitors have been shown to induce apoptosis [11] in pancreatic cancer cell lines as well as potentiate the growth inhibitory effects of chemotherapeutic agents including gemcitabine $[6,12]$. Celecoxib, a selective COX-2 inhibitor, inhibited the growth of human pancreatic cancer cell lines [13]. Clinical trials suggested an improved safety profile of celecoxib in comparison to non-selective COX inhibitors with respect to gastrointestinal and renal toxicity $[14,15]$.

Gemcitabine is the most commonly used cytotoxic agent in advanced pancreatic cancer [2]. However, 
objective response rates of patients with pancreatic cancer treated with this drug are less than $10 \%$ without clinically significant impact on survival (6 month and median survival of $46 \%$ and 5 to 6 months, respectively) [16]. Gemcitabine is therefore combined with other cytotoxic drugs to improve its efficacy in this disease. Phase II trials of gemcitabine and cisplatin in advanced pancreatic cancer have demonstrated objective response rates of 11 and $31 \%$, 6 month and median survival of $64 \%$ and 7 to 8 months, respectively $[17,18]$. Subsequently, two Phase III studies comparing the gemcitabine and cisplatin combination to gemcitabine were conducted $[19,20]$. The median time to progression and the overall response rate were significantly higher in the combination arms. However, the survival duration, though longer with the combination therapy, did not achieve statistical significance. These trials utilized the conventional short infusion (30-45 minutes) schedules of gemcitabine. A second approach to improve the efficacy of gemcitabine was based on improving its cellular activation to deoxycytidine triphosphate. The cellular uptake and activation of gemcitabine was saturable and hence infusion dose rate dependent. An optimized fixed dose infusion schedule of gemcitabine was shown to increase the intracellular accumulation of deoxycytidine triphosphate [21]. A randomized phase II trial comparing the fixed dose rate (FDR) infusion schedule of gemcitabine to bolus administration yielded a higher objective response rate and 1-year survival with the FDR [22]. Therefore, the FDR schedule of gemcitabine in combination with cisplatin could provide an active cytotoxic regimen that would be further enhanced by incorporating a targeted agent such as celecoxib.

We hypothesized that COX-2 inhibition by celecoxib would significantly improve the outcome of patients with metastatic pancreatic cancer treated with combination chemotherapy. To test this hypothesis we treated patients with metastatic pancreatic cancer with the triple drug combination of gemcitabine by fixed dose infusion, cisplatin, and celecoxib. The primary endpoint of the study was survival at 6 months.

\section{Materials and methods}

\section{Patient eligibility}

Patients with a histologic or cytologic diagnosis of pancreatic adenocarcinoma and metastatic disease were eligible. Patients were required to have a Southwest Oncology Group (SWOG) performance status of 0-2, a life expectancy of at least 3 months, and adequate hematologic, renal and hepatic function defined by the following parameters: neutrophil count $\geq 1,500 / \mathrm{mm}^{3}$, platelet count $\geq 100,000 / \mathrm{mm}^{3}$, creatinine $\leq 1.5 \mathrm{mg} / \mathrm{dL}$, total serum bilirubin $\leq 3.0 \mathrm{mg} / \mathrm{dl}$ and aspartate transaminase (AST) less than 3 times upper limit of the institutional normal range (ULN). Patients were excluded if they had neuroendocrine cancer or other active malignancy within the preceding year except for adequately treated basal cell, squamous cell skin cancer, or in-situ cervical cancer. Additional exclusion criteria included active peptic ulcer disease within the preceding year, and previous chemotherapy for pancreatic cancer except for patients relapsing more than 6 months after completion of adjuvant chemotherapy. All patients provided a signed informed consent in accordance with the Institutional Human Investigation Committee guidelines prior to enrolment on the study.

\section{Study design and treatment plan}

Gemcitabine (Gemzar, Eli Lilly, Indianapolis, IN) at a dose of $1,000 \mathrm{mg} / \mathrm{m}^{2}$ was administered intravenously on days 1 and 8 of each treatment cycle at a rate of $10 \mathrm{mg} / \mathrm{m}^{2} /$ minute. Cisplatin $35 \mathrm{mg} / \mathrm{m}^{2}$ was given intravenously on days 1 and 8 following gemcitabine infusion and with adequate hydration. Celecoxib (Celebrex, Pfizer Crop., New York, NY) was administered orally at a dose of $400 \mathrm{mg}$ twice daily. In order to achieve steady state levels prior to initiation of chemotherapy, celecoxib was started on day -7 . Celecoxib was continued until the end of study participation. Treatment cycles were repeated every 21 days.

Dose reductions were made on day 8 based on the toxicity encountered within that cycle. Doses of gemcitabine, celecoxib and cisplatin were held for a neutrophil count less than $500 / \mathrm{mm}^{3}$ or platelets less than $50,000 / \mathrm{mm}^{3}$ on the day of treatment. The gemcitabine and cisplatin doses were reduced by $20 \%$ for any grade 3 hematologic toxicity. All three drugs were held for grade 3 or 4 nonhematologic toxicity until recovery to grade 1 or lower. Dose adjustments on day 1 of a cycle were based on the worst grade of toxicity in the preceding cycle as follows: patients who sustained either febrile neutropenia or grade 4 myelosuppression received gemcitabine and cisplatin at $60 \%$ of the commencing dose of the previous cycle. For grade 3 and 4 non-hematologic toxicities excluding alopecia, nephrotoxicity, nausea, or vomiting the doses of gemcitabine and cisplatin were reduced by $20 \%$. Treatment for patients with renal toxicity was guided by the serum creatinine on the day of cisplatin administration and the extent of renal toxicity in the prior cycle. Patients with a serum creatinine of 1.6-2.0 times X ULN had a dose reduction of cisplatin by $40 \%$. Patients with a serum creatinine $\geq 2.1 \times$ ULN had the cisplatin dose held. Cisplatin dose was also adjusted based on the peak creatinine level during the previous cycle. For a creatinine of 2.1$3.0 \times$ ULN, the cisplatin dose was reduced by $25 \%$ if the 
serum creatinine level at the next dose was $\leq 1.5 \times$ ULN. Patients with a peak serum creatinine level of 3.1-4.5x ULN had the subsequent dose of cisplatin reduced by $50 \%$ if the serum creatinine level on the day of the next treatment was $\leq 1.5 \times$ ULN. When the serum creatinine level was $>4.5 \times$ ULN, no further cisplatin was given. Celecoxib was withheld for patients who developed peptic ulcer disease or a rise in serum creatinine level. The celecoxib dose was not adjusted for other toxicities. Once the dose of any drug was reduced during a treatment cycle, re-escalation was not permitted in subsequent cycles.

A new cycle of therapy could only begin if the neutrophil count was $\geq 1,500 / \mathrm{mm}^{3}$, platelet count was $\geq 100,000 / \mathrm{mm}^{3}$ and all relevant non-hematological toxicities were grade 1 or lower. Patients requiring a delay in therapy of longer than 2 weeks because of ongoing toxicities or more than two dose reductions were removed from the study. In addition, patients were removed from the study for any of the following reasons: disease progression, unacceptable toxicity, or withdrawal of consent.

\section{On-study evaluation}

Standard efficacy endpoints of objective response, time to progression, and survival were assessed. Imaging studies were performed at baseline and repeated after every 2 cycles of therapy or whenever there was any clinical suspicion of disease progression. Tumor responses were determined by the RECIST criteria and categorized as complete response, partial response, disease progression, or stable disease. Objective responses required at least one additional confirmatory follow up scan at least 3 weeks after the documentation of response. CA 19-9 was measured at baseline and after each cycle of treatment. Response by CA19-9 was defined as a decrease in CA 19-9 of at least $20 \%$ after the first cycle of treatment. Survival was measured from study registration to date of death or last follow up. Time to progression (TTP) was measured from study registration to the date of first documented progressive disease, or death. Time to treatment failure (TTF) was measured from study registration to the date of first documented progressive disease, or date off treatment due to toxicity, patient refusal, or death, whichever occurred first. Toxicities were evaluated at a minimum on days 1 and 8 of each cycle and graded according to National Cancer Institute Common Toxicity Criteria (NCI-CTC) version 2.0.

\section{Statistical methods}

This phase II trial was planned with a Simon two-stage optimal design [23]. Survival for at least 6 months after the date of registration was considered a treatment success. We wished to distinguish these regions of the true, unknown success rate: at least 0.60 versus at most 0.80 . The 2-stage design called for a maximum of 28 evaluable patients, 17 in Stage 1 and 11 in Stage 2. Patients were considered evaluable for the primary endpoint (6-month survival, yes/no) if they had completed at least two cycles of therapy. The design had a type I error of 0.142 and power of 0.901 . We needed at least 11 successes (i.e., 6-month survivors) among the first 17 evaluable patients to justify beginning Stage 2 of the study. After accruing 22 patients (over 16 months), there were 17 evaluable patients. With only 9 confirmed 6-month survivors in Stage 1 of the trial, we concluded that the sample success proportion $(9 / 17=53 \%)$ better supported the null hypothesis that the true, unknown success rate was at most 0.60 .

Exact, minimum-width $90 \%$ confidence intervals (CI) for response and toxicity rates were calculated using the Casella method [24] as implemented in StatXact software [25]. Standard Kaplan-Meier estimates of the censored response duration and censored survivorship functions were computed. Due to the small sample sizes, survival statistics (e.g., median) were estimated more conservatively using linear interpolation [26] among successive event times on the Kaplan-Meier curves.

\section{Results}

\section{Patient characteristics}

Patient characteristics are outlined in Table 1.Twenty-two patients were enrolled onto the study between January 2002 and May 2003. Data was collected until February 25, 2004. The median age at study entry was 59.5 years (range 41-77 years). There were proportionally more males (59\%). Eighty-two percent of the patients had a performance status of 1 . Twenty patients had liver

Table 1. Characteristics of the 22 patients with advanced pancreatic cancer treated with gemcitabine, cisplatin and celecoxib

\begin{tabular}{lrrl}
\hline Characteristics & Number & Percentage & Years \\
\hline Median age & & & $59.5(41-77)$ \\
Sex & & & \\
$\quad$ Male & 13 & 59.1 & \\
$\quad$ Female & 9 & 40.9 & \\
Race & & & \\
$\quad$ African-American & 3 & 13.6 & \\
$\quad$ Caucasian & 17 & 77.3 & \\
$\quad$ Other & 2 & 9.0 & \\
Performance status & & 13.6 & \\
$\quad 0$ & 3 & 81.8 & \\
$\quad 1$ & 18 & 4.5 & \\
$\quad 2$ & 1 & 86 & \\
Metastatic Site & & 32 & \\
$\quad$ Liver & 19 & 7 & \\
$\quad$ Peritoneum & &
\end{tabular}


metastases and two patients had only peritoneal metastases. No patient received prior chemotherapy or radiation treatment.

\section{Treatment administration}

A total of 91 cycles were administered with a median of 2 cycles per patient (range 0 to 11 ). Seventeen patients received two or more cycles of therapy and were evaluable for the primary endpoint. Two patients received no therapy for the following reasons: one patient was lost to follow up after registration, and one did not meet the eligibility criteria. Three patients received only day 1 of cycle 1 for the following reasons: myocardial infarction, bacterial peritonitis, and rapid disease progression. Fifteen of seventeen patients required dose reductions. The most common causes for dose reduction were neutropenia (9 patients), thrombocytopenia (3 patients), and renal toxicity (3 patients).

\section{Survival}

Survival and response were analyzed on an intention to treat basis. The Kaplan- Meier estimate of survival for the
22 patients is given in Figure 1. The median survival time was 5.8 months (90\% CI, 3.6-7.6 months). The 6-month survival rate was $46 \%$ (90\% CI, 27-62\%). Two patients are still alive (at 9.9 and 12.3 months) for a censoring rate of $9.1 \%$.

\section{Objective response, $T T F, T T P$}

Three patients had a partial response (PR) and 7 patients had stable disease (SD). The PR and SD rates were 14\% (90\% CI, 0.05-0.31) and 32\% (90\% CI, 0.18-0.50), respectively. Two of the three responders have progressed after 5 and 6 months from the time of documented response. One patient was still free of progression at 7.3 months. The baseline CA19-9 was available on 18 of the 22 patients. After the first cycle, CA 19-9 was available on 11 patients. A $20 \%$ decrease in CA 19-9 was observed in 5 of the 11 patients accounting for a response rate by CA19-9 of $45.5 \%$.

The median time to treatment failure was 1.3 months (90\% CI, 1.1-3.7 months). The median time to progression was 1.8 months (90\% CI, 0.4-3.1 months). The Kaplan-Meier estimate of the time to progression curve is given in Figure 2.

\section{Overall Survival}

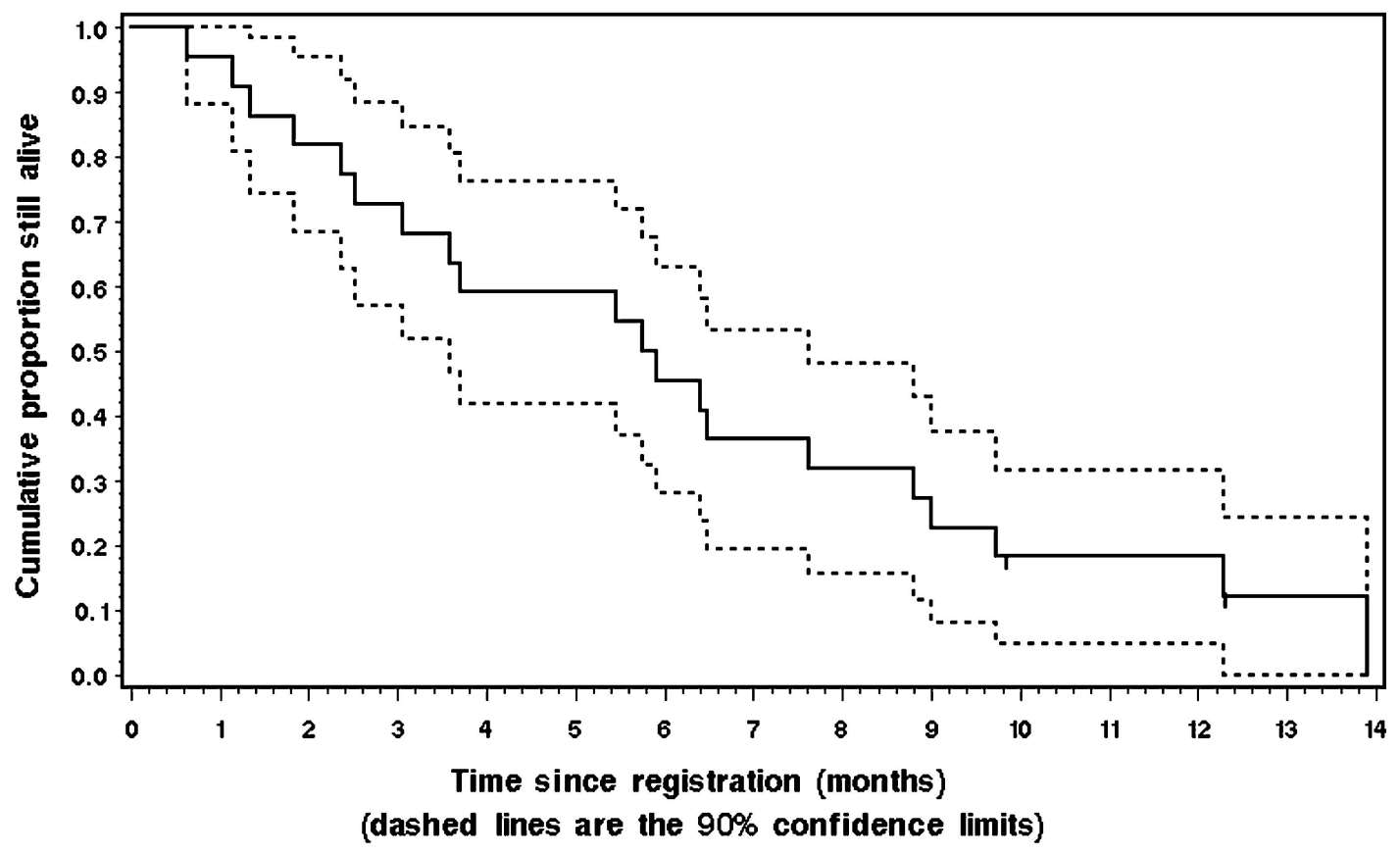

Patients

$\begin{array}{llllllllllllllllllll}\text { at risk } & 22 & 21 & 18 & 16 & 13 & 13 & 2 & 8 & 7 & 6 & 3 & 3 & 1 & 0\end{array}$

Figure 1. The Kaplan-Meier estimate of overall survival in the 22 patients with metastatic pancreatic cancer enrolled on the study. The dashed lines represent the $90 \%$ confidence interval (CI) about each successive estimate of the survival rate. The median survival was 5.8 months (90\% CI, 3.6-7.6 months). The 6-month survival rate was $45 \%$ (90\% CI, 27-62\%). 
Time to Progression

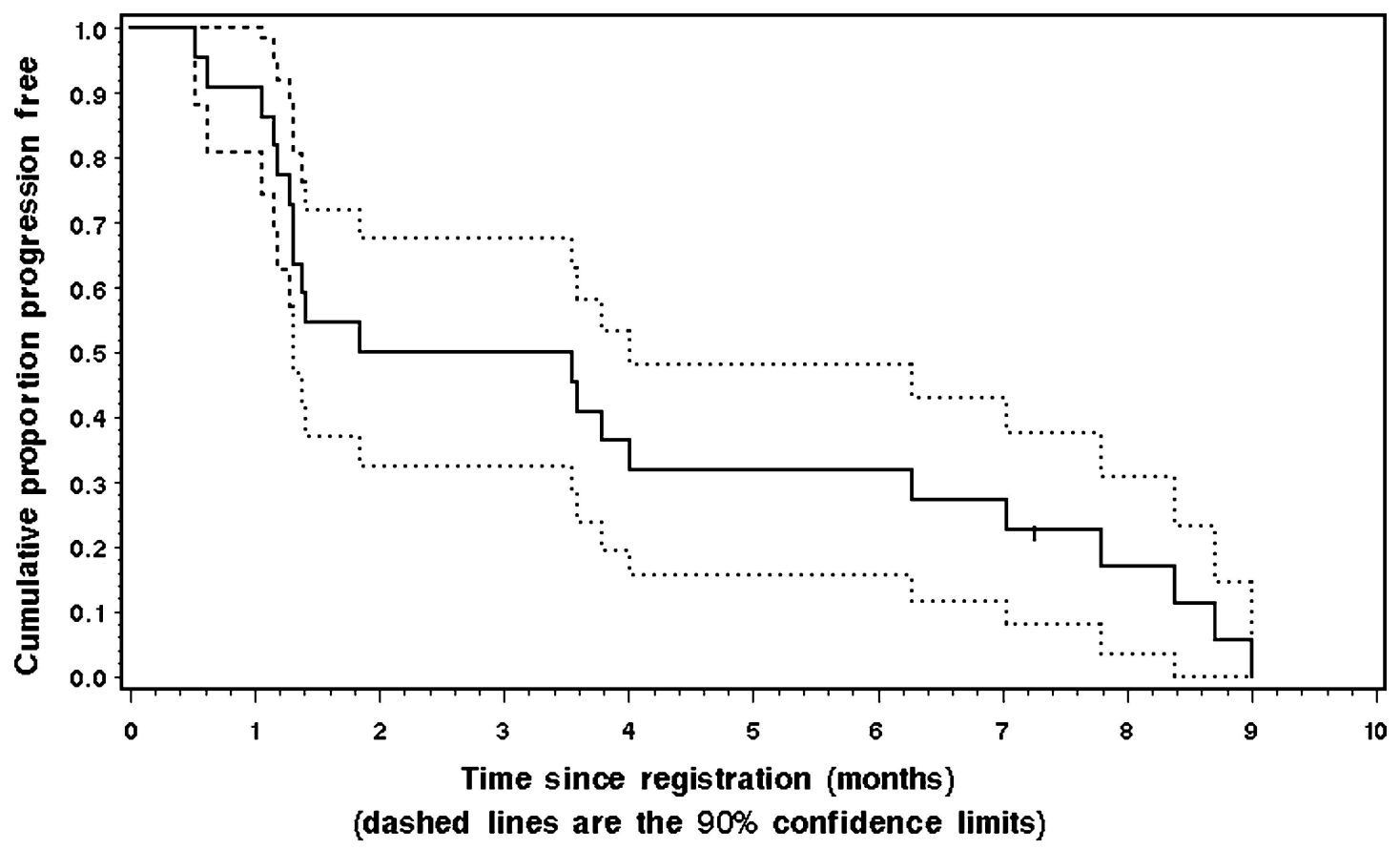

Patients

$\begin{array}{llllllllll}\text { at risk } & 22 & 20 & 11 & 11 & 8 & 7 & 7 & 6 & 1\end{array}$

Figure 2. The Kaplan-Meier estimate of time to progression in the 22 patients with metastatic pancreatic cancer who were treated with gemcitabine, cisplatin and celecoxib. The dashed lines represent the $90 \%$ confidence interval (CI) about each successive estimate of the progression-free rate. The median time to progression was 1.8 months (90\% CI, 0.4-3.1 months).

\section{Toxicity}

Two patients did not receive any treatment and could not be evaluated for toxicity. The treatment was generally well tolerated in the outpatient setting. No treatment-related deaths were reported. Table 2 summarizes the grade 3 and 4 toxicities observed on the study. Seven episodes of grade 4 toxicities were observed in 6 patients. The most common grade 3-4 toxicities were hematologic. Thirteen patients had grade 3-4 neutropenia. Infections were observed in four patients. One patient had a grade 3 febrile neutropenia, and three patients had non-neutropenic infections ( 1 grade 3 bacterial peritonitis and 2 grade 2 infections). Nine patients had grade 3 thrombocytopenia. Three of these patients developed bleeding on the study (1 grade 3 GI bleeding source unspecified, 1 grade 3 upper GI bleeding due to esophageal varices, and 1 grade 2 vaginal bleeding). Nine patients required hospitalization (3 cardiac events, 2 dehydration and anemia, and one each for neutropenic fever, bacterial peritonitis with GI bleeding, biliary stent replacement, and diarrhea).

Grade 2 asthenia was observed in 7 patients. Three patients had grade 2 renal toxicity requiring dose reductions of cisplatin. Three patients developed cardiac events while on treatment. The first patient who was known to have a history of atrial tachyarrhythmia developed atrial flutter during the second cycle of therapy. The second patient who was known to have stable coronary artery disease developed unstable angina during the first cycle

Table 2. The frequency of grade 3-4 treatment related toxicities in 20 patients with pancreatic cancer expressed as the worst toxicity per patient. Toxicity was assessed using the NCI-CTC version 2.0 scale for toxicity grading

\begin{tabular}{|c|c|c|c|c|}
\hline \multirow[b]{2}{*}{ Type of toxicity } & \multicolumn{2}{|c|}{ Grade 3} & \multicolumn{2}{|c|}{ Grade 4} \\
\hline & Number & Percentage & Number & Percentage \\
\hline Neutropenia & 10 & 50 & 3 & 15 \\
\hline Leukopenia & 3 & 15 & 1 & 5 \\
\hline Thrombocytopenia & 9 & 45 & 0 & 0 \\
\hline Anemia & 6 & 30 & 1 & 5 \\
\hline Infection & 2 & 30 & 0 & 0 \\
\hline Angina & 1 & 5 & 1 & 5 \\
\hline Arrhythmia & 1 & 5 & 0 & 0 \\
\hline Bleeding & 1 & 5 & 1 & 5 \\
\hline Dehydration & 2 & 10 & 0 & 0 \\
\hline Diarrhea & 2 & 10 & 0 & 0 \\
\hline Nausea & 4 & 20 & 0 & 0 \\
\hline Vomiting & 3 & 15 & 0 & 0 \\
\hline
\end{tabular}


of therapy. This patient was hospitalized and underwent a cardiac catheterization, which revealed extensive coronary artery disease. The patient was managed medically and received 10 additional cycles of therapy with no further cardiac events. The third patient who was not known to have any previous cardiac disease developed a myocardial infarction after receiving day 1 of cycle 1 . The patient subsequently had a cardiac catheterization, which revealed extensive coronary artery disease and was removed from the study.

\section{Discussion}

The COX-2 enzyme is a modulator of carcinogenesis, apoptosis, and angiogenesis in pancreatic cancer. In preclinical models of human pancreatic cancer, inhibition of the COX-2 enzyme potentiated the activity of gemcitabine $[6,11,13]$. Based on this evidence, we hypothesized that combining celecoxib with cytotoxic chemotherapy would result in an improvement in survival of patients with metastatic pancreatic cancer. The rationale for the cytotoxic regimen was based on the results of two phase III trials demonstrating a higher response rate and longer time to progression with gemcitabine and cisplatin as compared to single agent gemcitabine $[19,20]$ in those trials a trend towards improvements in survival was noted that was statistically insignificant. To further improve the activity of gemcitabine, a fixed dose rate infusion schedule that has been shown to increase the intracellular activation of gemcitabine was used. The regimen used in this study was therefore a modification of previous dose and schedules of gemcitabine and cisplatin combinations. The modifications were based on the dose intensity of cisplatin that was feasible in previous studies. The rationale for the dose of celecoxib used in this study was the demonstrated activity of celecoxib at $800 \mathrm{mg} /$ day to inhibit intratumoral activity of the COX-2 enzyme in non-small cell lung cancer [27], and the published benefit of this dose level in the prevention of colorectal polyp formation in familial adenomatous polyposis [28].

In the present study, the triple combination therapy of gemcitabine, cisplatin, and celecoxib resulted in a 1-year survival rate of $13 \%$ and a median survival of 5.8 months in patients with metastatic pancreatic cancer. These results are comparable to the previously reported survival rates observed with single agent gemcitabine [16] administered by a 30-45 minute infusion schedule. Several possible interpretations for the observed results could be entertained. First, COX-2 inhibitors may arrest cells at the $\mathrm{G}_{1}-\mathrm{S}$ phase [12] and therefore antagonize the effects of $S$ phase specific agents such as gemcitabine. A pulsatile schedule of administration of celecoxib prior to chemotherapy could potentially decrease the cell cycle effects of celecoxib while maintaining its pro-apoptotic effects. Such a schedule may be evaluated initially in preclinical models of human pancreatic cancer. Second, recent data from preclinical models suggest that nimesulide, a selective COX-2 inhibitor, can stimulate angiogenesis and growth of pancreatic cancer cell lines that do not express the COX-2 enzyme [29]. Conversely, the pro-apoptotic effects of celecoxib may be best demonstrated in cells overexpressing COX-2. In this study, no evaluation of baseline tumoral COX-2 expression was performed because of a lack of a reliable assay. Future studies may address the benefits of combining celecoxib with cytotoxic therapy only in patients whose tumors overexpress the COX-2 enzyme. Third, the increased hematologic toxicity with the current regimen required dose reductions in the majority of the patients on the trial. The impact of the dose reduction might have attenuated any potential benefit from combining celecoxib with the gemcitabine plus cisplatin regimen. Alternatively, the inhibition of the COX-2 enzyme alone maybe insufficient to reverse the chemoresistance in pancreatic cancer. Given some of the redundancies of the cell signalling pathways, combinations of COX-2 inhibitors and other targeted molecules may be necessary to significantly sensitize pancreatic cancer cells. Ongoing trials evaluating the effects of combining celecoxib with gemcitabine [30] or gemcitabine and irinotecan [31] may further define the role of celecoxib in the treatment of pancreatic cancer.

Preclinical observations suggested that COX-2 inhibition may delay the recovery phase of chemotherapyinduced myelosuppression [32]. An increase in myelotoxicity was also observed when celecoxib was combined with paclitaxel and carboplatin in patients with non-small cell lung cancer [27]. In our study, the incidence of grade 3-4 neutropenia (65\%) was slightly higher than that reported by Ko et al. (57\%) with gemcitabine by fixed-dose rate infusion and cisplatin [33].

Cardiovascular morbidity remains a concern with longterm use of selective COX-2 inhibitors. In this trial, three patients experienced cardiac events while on treatment. All those patients had established pre-treatment cardiac disease. Blanke et al. reported a similar increase in thrombotic episodes in patients with colorectal cancer receiving irinotecan, 5-fluorouracil, and celecoxib [34]. The increase in thrombotic complications could be due to the higher dose of celecoxib used or the hypercoagulable state of patients with metastatic cancer receiving chemotherapy.

In conclusion, the antitumor activity of gemcitabine by FDR and cisplatin was not improved with concurrent administration of the COX-2 inhibitor celecoxib. This lack of benefit of a COX-2 inhibitor does not rule out the significance of the COX-2 enzyme as a target for drug development in pancreatic cancer. Attempts to select 
patients who may respond to such therapy and the combination of COX-2 inhibitors with novel targeted agents would be of continued research interest.

\section{References}

1. Jemal A, Tiwari RC, Murray T, Ghafoor A, Samuels A, Ward E, Feuer EJ, Thun MJ, American Cancer Society: Cancer statistics, 2004. CA Cancer J Clin 54(1): 8-29, 2004

2. El-Rayes BF, Shields AF, Vaitkevicius V, Philip PA: Developments in the systemic therapy of pancreatic cancer. Cancer Invest 21(1): 73-86, 2003

3. Funk CD: Prostaglandins and leukotrienes: Advances in eicosanoid biology. Science 294(5548): 1871-1875, 2001

4. Ding XZ, Hennig R, Adrian TE: Lipoxygenase and cyclooxygenase metabolism: New insights in treatment and chemoprevention of pancreatic cancer. Mol Cancer 2(1): 10, 2003

5. Merati K, said Siadaty M, Andea A, Sarkar F, Ben-Josef E, Mohammad R, Philip P, Shields AF, Vaitkevicius V, Grignon DJ, Adsay NV: Expression of inflammatory modulator COX-2 in pancreatic ductal adenocarcinoma and its relationship to pathologic and clinical parameters. Am J Clin Oncol 24(5): 447-452, 2001

6. Yip-Schneider MT, Barnard DS, Billings SD, Cheng L, Heilman DK, Lin A, Marshall SJ, Crowell PL, Marshall MS, Sweeney CJ: Cyclooxygenase-2 expression in human pancreatic adenocarcinomas. Carcinogenesis 21(2): 139-146, 2000

7. Sheng H, Shao J, Washington MK, DuBois RN: Prostaglandin E2 increases growth and motility of colorectal carcinoma cells. J Biol Chem 276(21): 18075-18081, 2001

8. Gately S: The contributions of cyclooxygenase-2 to tumor angiogenesis. Cancer Metastasis Rev 19(1-2): 19-27, 2000

9. Araki Y, Okamura S, Hussain SP, Nagashima M, He P, Shiseki M, Miura K, Harris CC: Regulation of cyclooxygenase-2 expression by the Wnt and ras pathways. Cancer Res 63(3): 728-734, 2003

10. Sheng H, Shao J, Dubois RN: K-Ras-mediated increase in cyclooxygenase 2 mRNA stability involves activation of the protein kinase B1. Cancer Res 61(6): 2670-2675, 2001

11. Molina MA, Sitja-Arnau M, Lemoine MG, Frazier ML, Sinicrope FA. Increased cyclooxygenase-2 expression in human pancreatic carcinomas and cell lines: Growth inhibition by nonsteroidal antiinflammatory drugs. Cancer Res 59(17): 4356-4362, 1999

12. Yip-Schneider MT, Sweeney CJ, Jung SH, Crowell PL, Marshall MS: Cell cycle effects of nonsteroidal anti-inflammatory drugs and enhanced growth inhibition in combination with gemcitabine in pancreatic carcinoma cells. J Pharmacol Exp Ther 298(3): 976985, 2001

13. El-Rayes BF, Ali S, Sarkar FH, Philip PA: Cyclooxygenase-2dependent and -independent effects of celecoxib in pancreatic cancer cell lines. Mol Cancer Ther 3(11): 1421-1426, 2004

14. Whelton A, Fort JG, Puma JA, Normandin D, Bello AE, Verburg KM: Cyclooxygenase-2-specific inhibitors and cardiorenal function: A randomized, controlled trial of celecoxib and rofecoxib in older hypertensive osteoarthritis patients. Am J Ther 8(2): 85-95, 2001

15. Silverstein FE, Faich G, Goldstein JL, Simon LS, Pincus T, Whelton A, Makuch R, Eisen G, Agrawal NM, Stenson WF, Burr AM, Zhao WW, Kent JD, Lefkowith JB, Verburg KM, Geis GS: Gastrointestinal toxicity with celecoxib vs nonsteroidal anti-inflammatory drugs for osteoarthritis and rheumatoid arthritis: the CLASS study: A randomized controlled trial. Celecoxib Long-term Arthritis Safety Study. Jama 284(10): 1247-1255, 2000
16. Burris H, Storniolo AM: Assessing clinical benefit in the treatment of pancreas cancer: Gemcitabine compared to 5-fluorouracil. Eur J Cancer 33(Suppl 1): S18-22, 1997

17. Philip PA, Zalupski MM, Vaitkevicius VK, Arlauskas P, Chaplen R, Heilbrun LK, Adsay V, Weaver D, Shields AF: Phase II study of gemcitabine and cisplatin in the treatment of patients with advanced pancreatic carcinoma. Cancer 92(3): 569-577, 2001

18. Heinemann V, Wilke H, Mergenthaler HG, et al: Gemcitabine and cisplatin in the treatment of advanced or metastatic pancreatic cancer. Ann Oncol 11(11): 1399-1403, 2000

19. Colucci G, Giuliani F, Gebbia V, Bilietto M, Rabitti P, Uomo G, Cigolari S, Testa A, Maiello E, Lopez M: Gemcitabine alone or with cisplatin for the treatment of patients with locally advanced and/or metastatic pancreatic carcinoma: A prospective, randomized phase III study of the Gruppo Oncologia dell'Italia Meridionale. Cancer 94(4): 902-910, 2002

20. Heinemann V, Quietzsch D, Gieseler F, Gonnermann H. A phase III study comparin gemcitabine and cisplatin to gemcitaine alone in advanced pancreatic cancer. Paper presented at: American Society Clinical Oncology, 2003; Chicago, IL.

21. Touroutoglou N, Gravel D, Raber MN, Plunkett W, Abbruzzese JL. Clinical results of a pharmacodynamically-based strategy for higher dosing of gemcitabine in patients with solid tumors. Ann Oncol 9(9): 1003-1008, 1998

22. Tempero M, Plunkett W, Ruiz Van Haperen V, et al: Randomized phase II comparison of dose-intense gemcitabine: Thirty-minute infusion and fixed dose rate infusion in patients with pancreatic adenocarcinoma. J Clin Oncol 21(18): 3402-3408, 2003

23. Simon R. Optimal two-stage designs for Phase II clinical trials. Controlled Clinical Trials 10: 1-10, 1989

24. Casella G. Refining binomial confidence intervals. Canadian J. Statistics 14: 113-129, 1987

25. Mehta C, Patel N: StatXact 5: Statistical software for exact nonparametric inference, user manual: Cytel Software Corporation; 1999.

26. Lee E: Statistical Methods for Survival Data Analysis, 2nd ed. Wiley \& Sons, Inc., 1992.

27. Altorki NK, Keresztes RS, Port JL, et al: Celecoxib, a selective cyclo-oxygenase- 2 inhibitor, enhances the response to preoperative paclitaxel and carboplatin in early-stage non-small-cell lung cancer. J Clin Oncol 21(14): 2645-2650, 2003

28. Steinbach G, Lynch PM, Phillips RK, Wallace MH, Hawk E, Gordon GB, Wakabayashi N, Saunders B, Shen Y, Fijimura T, Su LK, Levin B: The effect of celecoxib, a cyclooxygenase-2 inhibitor, in familial adenomatous polyposis. N Engl J Med 342(26): 1946-1952, 2000

29. Ebil G, Reber H, Okada Y, Rix T, Hines O: The selective COX-2 inhibitor nimesulide stimulates angiogenesis and growth of COX-2 negative pancreatic cancer in vivo. Paper presented at: Gastroinestinal Cancer Symposium; January 22-24, 2004; San Franscisco, Ca.

30. Smith SE, Burris HA, 3rd, Loehrer PJ, Sr., Sweeny C: Preliminary report of a Phase II trial of gemcitabine combined with celecoxib for advanced pancreatic cancer. Paper presented at: American Society Clinical Oncology; May 31-June 3, 2003; Chicago, Il.

31. Lipton A, Harvey H, Witters L, Kerr S, Legore K, Campbell C: Phase II trial of gemcitabine+irinotecan+celecoxib in pancreatic cancer. Paper presented at: Gastrointestinal Cancer Symposium; January 22-24, 2004; San Franscisco, CA.

32. Lorenz M, Slaughter HS, Wescott DM, Carter SI, Schnyder B, Dinchuk JE, Car BD: Cyclooxygenase-2 is essential for normal recovery from 5-fluorouracil-induced myelotoxicity in mice. Exp Hematol 27(10): 1494-1502, 1999

33. Ko A, Dito E, Schillinger B, Venook AP, Bersgland EK, Allen JN, Tempero MA: A phase II trial of gemcitabine given at fixed dose infusion in combination with cispplatin for metastatic 
adenocarcinoma of pancreas. Paper presented at: Gastrointestinal Cancers Symposium; January 22-24, 2004; San Franscisco, CA.

34. Blanke CD, Benson AB III, Dragovich T, Lenz H, Haller D, Tobles C, Buchbinder A: A phase II trial of celecoxib (CX), irinotecan (I), 5-Fluorouracil (5FU), and leucovorin (LCV) in patients (pts) with unresectable or metastatic colorectal cancer (CRC). Paper presented at: ASCO proceedings; May 18-21, 2002; Orlando, FL.

Address for offprints: Basil F. El-Rayes, MD, Karmanos Cancer Institute, Wayne State University, 4100 John R Street, Detroit, MI 48201. Tel.: 313745 8029; Fax: 313993 2944; E-mail: elrayesb@karmanos.org 